found in nutrient intake were independent of measures of child deprivation and ethnicity.

Conclusion Children taking a packed lunch to school have a less nutritious diet on average over the whole day in terms of sugar, sodium and fibre. A good quality lunch is therefore an important factor for overall dietary quality in children. Policies to encourage parents and children to include nutritious foods such as fruit, vegetables and low fat starchy foods are needed to narrow the gap between school meals and packed lunches.

\section{OP84 DO SUPERMARKET INTERVENTIONS IMPROVE FOOD ACCESS, FRUIT AND VEGETABLE INTAKE AND BMI? EVALUATION OF THE PHILADELPHIA FRESH FOOD FINANCING INITIATIVE}

doi:10.1136/jech-2012-201753.084

'E Flint, 'S Cummins, ${ }^{2}$ SA Matthews. 'School of Geography, Queen Mary University of London, London, UK; ${ }^{2}$ Department of Sociology, Pennsylvania State University, PA, USA

Background Structural interventions to improve access to healthy food in deprived communities are a current policy priority. In 2010, the Obama administration unveiled the Healthy Food Financing Initiative to promote interventions that expand access to nutritious foods. The initiative provided more than $\$ 400$ million in funding to bring grocery stores and health food retailers to deprived communities. However, no evidence for the effectiveness of such policy interventions exists. This paper is a first attempt to evaluate the effect of this intervention on perceptions of the food environment, fruit and vegetable intake and body mass index (BMI) in Philadelphia, USA.

Methods Data from the Philadelphia Neighbourhood Food Environment Study were used. This study utilised a prospective quasiexperimental design comparing baseline and follow up data in an 'intervention' community with a matched 'comparison' community. The intervention was the introduction of a large food supermarket in an underserved area. Outcomes were BMI, weekly fruit and vegetable consumption (Block FFQ), and perception of the neighbourhood food environment. Data were analysed using an intention-to-treat (ITT) and adopters vs non-adopters (ANA) approach. Difference-in-differences analyses (DID) assessed whether significantly different changes over time existed between intervention and comparison groups. Models were adjusted for age, sex, income, race, education, household composition and employment status.

Results In ITT and ANA analyses no significant difference-indifferences in mean BMI and mean weekly fruit and vegetable consumption were found. ITT analyses demonstrated a significant improvement in perceptions of the neighbourhood food environment in the intervention versus comparison neighbourhood (DID=1.71, $\mathrm{p}=0.001)$. Using ANA, a similar result was found for adopters, with those who used the grocery store as their primary shop (DID=1.57, $\mathrm{p}=0.05)$ and those who used it for any food shopping ( $\mathrm{DID}=2.34, \mathrm{p}<0.001$ ) having significantly better perceptions of the neighbourhood food environment compared to nonadopters. After adjustment, ITT analyses remained significant $(\mathrm{DID}=1.65, \mathrm{p}=0.002)$. For ANA, significant DID results were attenuated for primary adopters ( $\mathrm{DID}=1.30, \mathrm{p}=1.105$ ) but remained significant for those undertaking any food shopping (DID=2.18, $\mathrm{p}<0.001$ ).

Conclusion The introduction of a new grocery store had a positive effect on how local residents perceive the quality of their neighbourhood food environment. However, this did not have a significant effect on fruit and vegetable consumption or BMI. Improvement in perceptions of neighbourhood food environment quality did not translate into improved dietary behaviour or reductions in BMI.

\section{Population Based Studies: Mid Life and Older Age}

\section{OP85 DO GO0D HEALTH AND MATERIAL CIRCUMSTANCES PROTECT OLDER PEOPLE FROM THE INCREASE IN MORTALITY AFTER BEREAVEMENT?}

doi:10.1136/jech-2012-201753.085

'S M Shah, II M Carey, 'T Harris, 'S DeWilde, ${ }^{2} \mathrm{C}$ R Victor, 'D G Cook. 'Population Health Sciences and Education, St George's University of London, London, UK; ${ }^{2}$ School of Health Sciences and Social Care, Brunel University, Uxbridge, UK

Background Death of a spouse or partner is a common major life event for older people. The adverse health effects of bereavement are well recognised with an increased risk of death described in several populations. The impact of modifying factors, such as chronic disease and material circumstances, is less well understood. In this study, we use a large UK primary care database to examine the modifying and mediating effect of physical comorbidity and material socio-economic circumstances on the rise in mortality in the first year after bereavement.

Methods We identified 171,120 older (60 years and over) couples in a UK primary care database (THIN) based on a shared household identifier. The couples were followed up between 2005 and 2010 for an average of 4 years. 26,646 (15.5\%) couples experienced bereavement with mean follow up after bereavement of 2 years. The effect of bereavement on risk of death in the surviving partner was examined in a survival model adjusted for age, sex, comorbidity at baseline, material deprivation based on area of residence, season and smoking. Further analysis examined the effect of changes in comorbidity during follow up.

Results The fully adjusted hazard ratio (HR) for bereavement in the first year after bereavement was 1.25 (95\% CI: 1.18 to 1.33). Further adjustment for changes in comorbidity throughout follow up did not alter the hazard ratio for bereavement (HR 1.27, 95\% CI: 1.19 to 1.35). The effect of bereavement was not modified by age, gender or baseline comorbidity. The relative rise in mortality after bereavement was greatest in individuals with no significant chronic comorbidity throughout follow up (HR 1.50, 95\% CI: 1.28 to 1.77 ) and in more affluent couples $(P=0.035)$.

Conclusion We have confirmed the increased risk of mortality after bereavement and demonstrated its independence of pre-existing physician recorded chronic comorbidity and social status. Our analysis, taking account of changes in morbidity before and after bereavement, suggests that the rise in mortality after bereavement is not primarily mediated through new or worsening chronic physical disease. Furthermore, there was no evidence that pre-existing or continuing good health or affluence protect individuals. The results also suggest that, paradoxically, good health and high social status may accentuate the rise in mortality after bereavement. Our findings suggest that the rise in mortality after bereavement acts as a leveller, affording no protection to the affluent or healthy, and is best explained by an increase in sudden unexpected deaths.

\section{OP86 MORE RAPID DECLINE IN CHD INCIDENCE AMONG SCOTTISH TOWNS THAN AMONG ENGLISH TOWNS FROM THE 1980s TO THE 2000s}

doi:10.1136/jech-2012-201753.086

${ }^{1}$ E Cecil, 'SL Hardoon, 'SG Wannamethee, $2 \mathrm{PH}$ Whincup, 'RW Morris. 'Primary Care \& Population Health, UCL Medical School, London, UK; ${ }^{2}$ Population Health Sciences \& Education, St George's University of London, London, UK

Background The incidence of coronary heart disease (CHD) has declined in the UK since the late 1970s. However, regional differences in this decline are unknown. We investigate the change in 